\title{
Skeletal muscle insulin resistance in salt-sensitive hypertension: role of angiotensin II activation of $\mathrm{NF} K \mathrm{~B}$
}

\author{
Ming-Sheng Zhou ${ }^{1,3^{*}}$, Chang Liu², Runxia Tian ${ }^{3}$, Akira Nishiyama ${ }^{4}$ and Leopoldo Raij ${ }^{3,5}$
}

\begin{abstract}
Background: We have previously shown that in hypertensive Dahl salt-sensitive (DS) rats, impaired endotheliumdependent relaxation to acetylcholine and to insulin is mechanistically linked to up-regulation of angiotensin (Ang) II actions and the production of reactive oxygen species (ROS) and to activation of the proinflammatory transcription factor (NF)kB. Here we investigated whether Ang II activation of NFKB contributed to insulin resistance in the skeletal muscle of this animal model.
\end{abstract}

Methods: DS rats were fed either a normal (NS, $0.5 \% \mathrm{NaCl}$ ) or high ( $\mathrm{HS}, 4 \% \mathrm{NaCl})$ salt diet for 6 weeks. In addition, 3 separate groups of HS rats were given angiotensin receptor 1 blocker candesartan (ARB, $10 \mathrm{mg} / \mathrm{kg} /$ day in drinking water), antioxidant tempol (1 mmol/L in drinking water) or NFKB inhibitor PDTC (150 mg/kg in drinking water).

Results: DS rats manifested an increase in soleus muscle Ang II content, ROS production and phosopho-IkBa/lkBa ratio, ARB or tempol reduced ROS and phospho-IKBa/IKBa ratio. Hypertensive DS rats also manifested a reduction in glucose infusion rate, impaired insulin-induced Akt phosphorylation and Glut-4 translocation in the soleus muscle, which were prevented with treatment of either ARB, tempol, or PDTC. Data from the rat diabetes signaling pathway PCR array showed that 8 genes among 84 target genes were altered in the muscle of hypertensive rats with the increase in gene expression of ACE1 and 5 proinflammatory genes, and decrease of 2 glucose metabolic genes. Incubation of the muscle with NFKB SN50 (a specific peptide inhibitor of NFKB) ex vivo reversed changes in hypertension-induced gene expression.

Conclusion: The current findings strongly suggest that the activation of NFkB inflammatory pathway by Ang II play a critical role in skeletal muscle insulin resistance in salt-sensitive hypertension.

Keywords: Angiotensin II, Hypertension, Insulin resistance, NFKB, Skeletal muscle

\section{Introduction}

Hypertension and type 2 diabetic mellitus (T2DM) are two powerful risk factors for the development of cardiovascular diseases (CVD) [1,2]. Salt sensitivity of blood pressure and insulin resistance have been identified as key elements underlying the relationship between hypertension and T2DM. Excess dietary salt and caloric intake, as commonly found in westernized diets, are linked not only to increased blood pressure, but also to defective insulin sensitivity and impaired glucose homeostasis [3-5]. Insulin resistance is highly prevalent in the patients

\footnotetext{
*Correspondence: zhoums1963@163.com

'Department of Physiology, Liaoning Medical University, Jinzhou, China

${ }^{3}$ Hypertension/Nephrology Section, Miami VA Medical Center, Miami, FL, USA Full list of author information is available at the end of the article
}

with essential hypertension, particularly those with saltsensitivity [6]. However, there are still critical gaps in our knowledge of the mechanisms that lead to the development of insulin resistance in salt-sensitive (SS) hypertension.

Abnormal activation of renin-angiotensin system (RAS), oxidative stress, excessive dietary salt and fat intakes are factors that contribute to the development of insulin resistance [7-10]. Insulin stimulates glucose transport and vasodilation through the activation of phosphatidylinositol 3-kinase (PI3K) and nitric oxide (NO) signaling pathway $[11,12]$. It has been shown that angiotensin (Ang) II inhibits insulin stimulation of PI3K, thereby preventing the activation of downstream signaling molecules, including NO production in endothelial cells, and Glut-4 translocation in skeletal muscle cells $[13,14]$. We have recently 
shown in hypertensive Dahl SS (DS) rat, a paradigm of human SS hypertension characterized by cardiovascular injury and insulin resistance, that the upregulation of local (vasculature) Ang II activates the redox-sensitive transcription factor nuclear factor kappa (NFkB), which contributes to endothelial dysfunction, vascular inflammation, and the impairment of insulin-mediated vasorelaxation $[13,15,16]$.

There is considerable evidence showing that insulin resistance is associated with low-grade inflammation $[10,17-19]$. NF $\kappa B$ is a primary regulator for proinflammatory gene expression and inhibition of the NFKB inflammatory activation has been shown to improve insulin sensitivity and cardiovascular injury in metabolic and hypertensive diseases $[17,20]$. The skeletal muscle is the largest insulin sensitive tissue and handles over $75 \%$ of the insulin-mediated glucose disposal in the body [21]. Therefore, we tested the hypothesis that in SS hypertension, the activation of $\mathrm{NF \kappa B}$ inflammatory pathway by upregulation of endogenous Ang II/reactive oxygen species (ROS) impairs insulin sensitivity and insulin signaling in the skeletal muscle.

\section{Methods}

\section{Animals and experimental protocols}

The animals were housed in facilities accredited by the American Association for Accreditation of Laboratory Animal Care. The Institutional Animal Care and Use Committee at the Miami Veterans Affairs Medical Center approved the studies. Six-week-old DS male rats were purchased from Harlan Sprague-Dawley (Indianapolis, IN) and maintained under controlled conditions of light, temperature, and humidity. After 2 weeks of acclimatizing to the new environment, the rats were divided into 5 groups and treated for 6 weeks as follow: NS, fed $0.5 \%$ $\mathrm{NaCl}$ (normal salt) diet $(\mathrm{n}=6) ; \mathrm{HS}$, fed $4 \% \mathrm{NaCl}$ (high salt) $\operatorname{diet}(n=7) ; H S / A R B$ : fed HS plus angiotensin receptor 1 blocker candesartan (ARB, $10 \mathrm{mg} / \mathrm{kg} /$ day in drinking water, $n=7)$; HS/Temp: fed HS diet plus antioxidant tempol $(1 \mathrm{mmol} / \mathrm{L}$ in drinking water, $\mathrm{n}=6)$; HS/PDTC, fed HS diet plus pyrrolidine dithiocarbamate (PDTC, $150 \mathrm{mg} /$ $\mathrm{kg}$ in drinking water, $\mathrm{n}=6$ ), an inhibitor of NFkB activation. At the end of the study, the rats were euthanized by decapitation and the soleus muscles were harvested for determination of Ang II content, superoxide $\left(\mathrm{O}_{2}^{-}\right)$production and PCR array gene expression.

\section{Determination of Ang II level}

Ang II content in the soleus muscle was measured using the method established by Nishiyama et al and quantified with a competitive single antibody radioimmunoassay [22], using rabbit anti-Ang II antibody (Peninsula, Belmont, CA) and monoiodinated ${ }^{125}$ I-labeled Ang II (Amersham, Arlington Heights, IL). All samples were assayed in duplicates, and mean values were plotted against a curve generated by the Ang II standard.

\section{Determination of $\mathrm{O}_{2}^{-}$generation}

The soleus muscle was isolated and cut into small pieces. Generation of $\mathrm{O}_{2}^{-}$in fresh tissue was determined by the chemiluminescence of lucigenin $(5 \mu \mathrm{M})$, as previously described [23], and the results were expressed as counts/ $\mathrm{min} / \mathrm{mg}$ dry tissue. The chemiluminescence of lucigenin has been validated as a method in our previous studies to measure $\mathrm{O}_{2}^{-}$[23].

\section{Hyperinsulinemic-euglycemic clamp study and determination of intra-arterial pressure}

A separate group of rats were used to determine mean arterial blood pressure (MAP) and glucose infusion rate, an index for metabolic insulin sensitivity, measured by the hyperinsulinemic-euglycemic clamp. The animals were fasted overnight before the experiments were conducted. The rats were anesthetized with pentobarbital sodium (50 mg/kg I.P.) and maintained at $37^{\circ} \mathrm{C}$ with a heating pad. The right femoral vein and left femoral artery were catheterized and used for monitoring blood pressure. The arterial catheter was connected to a pressure transducer. After a 60-min equilibration period, MAP was recorded by Powerlab (ADInstruments Inc, Colorado Springs CO). The rats were then used for a hyperinsulinemic-euglycemic clamp study. The right femoral vein and left femoral artery were used for glucose and insulin infusion and blood sampling, respectively. Baseline plasma samples were obtained, after which insulin (Sigma) at a constant rate of $30 \mathrm{mU} \cdot \mathrm{kg}^{-1} \cdot \mathrm{min}^{-1}$ and glucose $(17.5 \mathrm{~g} / 100 \mathrm{ml}$ saline $)$ at varying infusion rates were continuously infused for $120 \mathrm{~min}$. The blood glucose concentration, measured with an automatic blood glucose meter (Accu-Chek Advantage Blood Glucose Meter), was clamped at $5.5 \mathrm{mmol} / \mathrm{L}$. Fasting plasma levels of insulin at baseline was measured by ELISA following the manufacture's instructions (R\&D Systems, Minneapolis, MN). Euglycemia was achieved by $60 \mathrm{~min}$ and maintained for $60 \mathrm{~min}$. The glucose infusion rate was adjusted according to the blood glucose levels at 5 -min intervals during the first 60 -min period and, once stable, at 15-min intervals during the second 60-min period. The samples obtained over the second 60-min period were averaged and reported as the mean steadystate glucose infusion rate $\left(\mathrm{mg} \cdot \mathrm{kg}^{-1} \cdot \mathrm{min}^{-1}\right)$ required for maintaining euglycemic conditions in the setting of hyperinsulinemia. After the completion of the euglycermic clamp, the soleus muscles were harvested and used for determination of the insulin signaling molecules.

\section{Western blot}

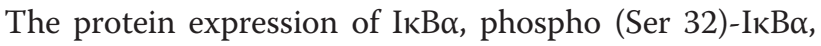
phospho (Ser 612)-IRS1, or phsopho (Ser 473)-Akt in 
the soleus muscle was determined by Western blot analysis. Briefly, after homogenization, protein concentration was determined by Bio-Rad protein assay. Thirty $\mu \mathrm{g}$ of protein were separated by SDS-PAGE and transferred to a nitrocellulose membrane. Transferred proteins were incubated overnight with specific polyclonal antibodies

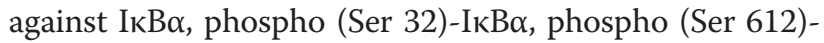
IRS1 or phospho (Ser 473)-Akt (Cell Signaling). After washing, the blots were incubated with a secondary antibody and the signal was detected by luminol chemiluminescence followed by exposure to an autoradiography film. The membrane was reblotted for $\beta$-actin (Santa Cruz Biotechnology Inc.), to serve as a loading control. The data was normalized to $\beta$-actin and expressed as fold increase versus NS group.

\section{Isolation of membrane fraction and determination of Glut-4 expression}

The soleus muscle was homogenized and centrifuged for $10 \mathrm{~min}$. at $1,000 \mathrm{~g}$ at $4^{\circ} \mathrm{C}$. The supernatant was saved; the pellet was resuspended in $1 / 3$ of the initial volume, and centrifuged again at $1,000 \mathrm{~g}$ for $10 \mathrm{~min}$. The two supernatant solutions were mixed and submitted to centrifugation at $41,000 \mathrm{~g}$ for one hour. The final pellet was resuspended in $100 \mu \mathrm{l}$ of buffer as a total membrane fraction. Glut-4 expression was determined by Western blot and normalized by $\beta$-actin.

\section{Real-time PCR}

Total RNA $(2 \mu \mathrm{g})$ was extracted from the soleus muscle and reverse-transcribed using the SuperScript II RT First Strand Synthesis kit (Gibco, BRL), according to the manufacturer's directions. Real-time PCR (RTq-PCR) for p22phox (Assay ID: Rn00577357_m1, Life Technologies), gp91phox (Assay ID: Rn00576710_m1) of nicotinamide adenine dinucleotide phosphate (reduced form, NADPH) oxidase subunits, suppressors of cytokine signaling 3 (SOCS3, Assay ID: Rn00585674-S1) and interleukin 6 (IL6, Assay ID:Rn00561420-m1) was performed in $20 \mu \mathrm{l}$ reaction mixture containing an appropriately diluted (80 ng) cDNA solution, $0.1 \mu \mathrm{mol} / \mathrm{L}$ each primer, $0.2 \mu \mathrm{mol} / \mathrm{L}$ probe and PCR Master Mix assay kit (ABI) as previously described [7]. All PCR primers with fluorescence probe were ordered through life technologies (ABI) online system with assay ID. The relative expression of each mRNA was normalized by a housekeeping gene (GADPH), and expressed as fold increase vs. the NS group.

\section{PCR array for determination of mRNA profile of rat diabetes signaling pathway in the soleus muscle} The soleus muscles from DS rats fed a NS or HS diet for 6 weeks were dissected and cut into small pieces. The muscles from HS rats were incubated with SN50 $(55 \mu \mathrm{g} / \mathrm{ml}$, a cell-permeable peptide specific inhibitor of NFkB nuclear translocation) or SM50 $(55 \mu \mathrm{g} / \mathrm{ml}$, a mutated inactive control peptide, BIOMOL Research Lab. Inc. (Plymouth Meeting, PA)) in DMEM containing 0.1\% BSA bubbled with $95 \%$ oxygen supplement for $120 \mathrm{~min}$. The muscle was homogenated in $1 \mathrm{ml}$ Trizol reagent (Life Technologies). One $\mu \mathrm{g}$ of RNA was converted to cDNA with random primers in a $20-\mu \mathrm{l}$ reaction volume using a high capacity cDNA archive kit (C-3, Superarray). The cDNA was diluted to a volume of $100 \mu \mathrm{l}$, one $\mu \mathrm{l}$ cDNA was used for each primer set in the PCR Array according to the manufacturer's protocol. Rat diabetes signaling pathway (PARN-023, Superarray) PCR array was used to determine a panel of diabetes gene expression. This PCR array includes 84 target genes related to obesity, insulin resistance, early onset of diabetes, complications from diabetes mellitus, and 12 control genes. Additionally, control genes are included in each array to control for genomic DNA contamination, RNA quality, housekeeping and general PCR performance. Data analysis was performed using the manufacturer's integrated web-based software package for the PCR Array System using deltadelta $\mathrm{Ct}$ based fold-change calculations and normalized by a housekeeping control gene. Based on PCR array results, we further confirmed the expression of SOCS3 and IL 6 by RTq-PCR.

\section{Data analysis}

The results were expressed as mean \pm standard error of the mean (SEM). Statistical analyses were performed by ANOVA with Bonferonni's correction for multiple comparisons. Significance was assumed at $\mathrm{p}<0.05$.

\section{Results}

\section{MAP and metabolic parameters}

High salt intake for 6 weeks significantly increased MAP (147 \pm 6 vs. $103 \pm 4 \mathrm{mmHg}$ in NS; $\mathrm{P}<0.05$ ), as assessed by direct intra-arterial measurements. ARB candesartan (HS/ARB), antioxidant tempol (HS/Temp) or PDTC (HS/PDTC) treatment slightly but significantly reduced MAP $(20-25 \%$ reduction, all $\mathrm{p}<0.05)$. The rats however remained severely hypertensive (Figure 1A). Hypertensive DS rats manifested a tendency towards decreased body weight gain as compared with NS rats, but this did not reach statistical significance $(384 \pm 13$ vs. $405 \pm 9 \mathrm{~g}$ in NS, $\mathrm{p}<0.1)$. The ARB, tempol or PDTC treatment did not affect body weight (data not shown). There were no significant differences in fasting plasma levels of insulin ( $3.6 \pm 0.9$ in HS vs. $3.3 \pm 0.7 \mathrm{ng} / \mathrm{ml}$ in NS, p >0.05) or glucose ( $95 \pm 6$ in HS vs. $91 \pm 5 \mathrm{mg} / \mathrm{dl}$ in NS, $\mathrm{p}>0.05$ ) between HS and NS DS rats. Treatment with ARB, tempol or PDTC did not affect fast plasma levels of insulin or glucose (data not shown). 

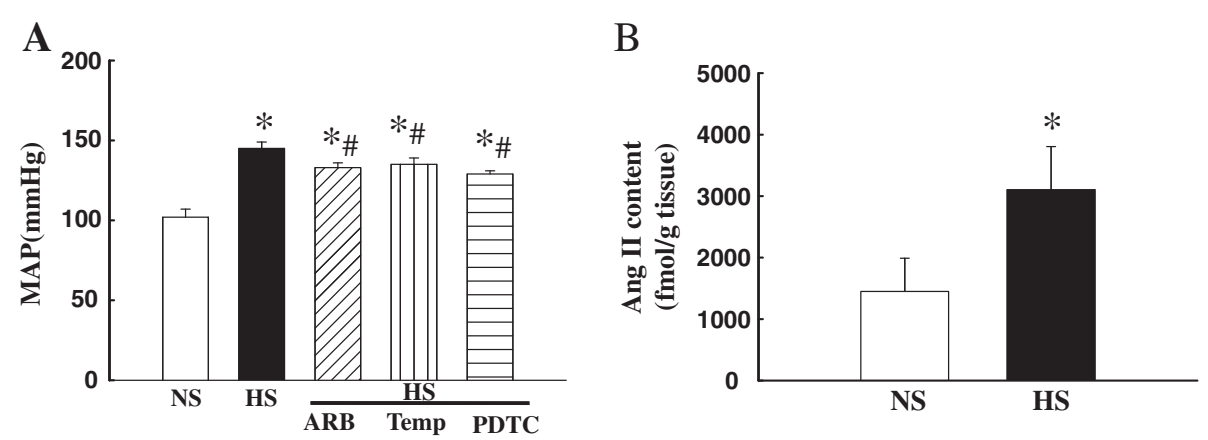

Figure 1 Mean arterial blood pressure (MAP, A) and Ang II content (B) in the soleus muscle of Dahl salt-sensitive (DS) rats. The data was expressed as mean \pm SEM. * $P<0.05$ vs. NS. \#p $<0.05$ vs. HS; $N=6-7$.

Increased endogenous Ang II level in the soleus muscle of hypertensive DS rats

As shown in Figure 1B, Ang II concentration in the soleus muscle from hypertensive DS rats was significantly higher than in those in the normotensive DS rats $(3145 \pm$ 575 vs. $1405 \pm 480 \mathrm{fmol} / \mathrm{g}$ tissue in NS, $\mathrm{p}<0.05$ ). To our knowledge, this is the first evidence showing an increase in endogenous Ang II level in the skeletal muscle of genetic SS hypertensive animal model.

Increased $\mathrm{O}_{2}^{-}$production was linked to upregulation of Ang II action in the soleus muscle of hypertensive DS rats We have previously shown that upregulation of Ang II was linked to increased vascular $\mathrm{O}_{2}^{-}$production in this animal model [16,23]. Hypertensive DS rats also had a significant increase in $\mathrm{O}_{2}{ }^{-}$production in the soleus muscle, determined by lucigenin chemiluminescence, and ARB candesartan normalized $\mathrm{O}_{2}^{-}$production (Figure $2 \mathrm{~A}$ ). In addition, using RTq-PCR, we determined the expression of NADPH oxidase subunits, including p22phox and gp91phox in the soleus muscle. The mRNA expression of p22phox and gp91phox in HS rats was significantly increased by $87 \%$ and $125 \%$ respectively, compared with NS rats (all $\mathrm{p}<0.05)$ and ARB treatment in HS-DS rats prevented the increase in the mRNA expression of $\mathrm{p} 22 \mathrm{phox}$ and gp91phox (all $\mathrm{p}<0.05$, Figure 2B-2C).

The treatment of ARB or tempol inhibited NFKB activation in the soleus muscle of hypertensive DS rats

$\mathrm{NF} K \mathrm{~B}$ activation is initiated from the phosphorylation of $I_{\kappa} B$. Decreased $I_{\kappa} B$ or increased ratio of phospho-I $\mathrm{K} B /$ I $K B$ is an index of NFKB activation [24]. As shown in Figure 3, hypertensive DS rats exhibited a significant decrease in $I_{\kappa} B \alpha$ expression and increase in the ratio of phospho-IkB $\alpha / \mathrm{IkB} \alpha$ compared with normotensive DS rats (all $\mathrm{p}<0.05)$. Either ARB candesartan or antioxidant tempol normalized $\mathrm{I} \kappa \mathrm{B} \alpha$ or the ratio of phsopho-IkB $\alpha /$ $\mathrm{IkB} \alpha$, suggesting upregulation of endogeous Ang II/ROS participate in $\mathrm{NF}_{\kappa} \mathrm{B}$ activation.
The ARB candesartan, antioxidant tempol or inhibition of NFKB PDTC improved insulin sensitivity in hypertensive DS rats

To determine the glucose infusion rate, an index of insulin sensitivity, we used the hyperinsulinemic-euglycemic clamp technique. As shown in Figure 4, the glucose infusion rate necessary to maintain plasma glucose levels at $5.5 \mathrm{mmol} / \mathrm{L}$ was significantly lower in HS rats when compared with NS rats $(\mathrm{p}<0.05)$, indicating the presence of metabolic insulin resistance in hypertensive DS rats. The treatment with either ARB, tempol or PDTC significantly improved the glucose infusion rate (all $\mathrm{p}<0.05)$.

\section{Protein expression of insulin signaling molecules}

It is well known that insulin induces glucose transport via activation of the IRS-1/PI3K/Akt/Glut-4 pathway [21]. The expression of phospho (Ser 612)-IRS-1 was significantly increased in HS group compared with NS group $(\mathrm{p}<0.05)$ and was normalized by the treatment of either ARB, tempol or PDTC treatment (Figure 5A). Downstream molecules of the insulin activation of the PI3K pathway in the skeletal muscle include the phosphorylation of Akt and Glut-4 translocation in the membrane. As shown in Figures $5 \mathrm{~B} \& \mathrm{C}$, the protein expression of phospho-Akt at Ser473, and Glut-4 in membrane fraction was significantly reduced in the soleus muscle of hypertensive DS rats (all $\mathrm{p}<0.05)$ and restored in the rats treated with either ARB, tempol or PDTC (Figure 5B\&C).

Effect of NFKB inhibition on gene profile related to onset, development and progression of diabetes in the soleus muscle of hypertensive DS rats

$\mathrm{NF} \kappa \mathrm{B}$ is a primary regulator of inflammatory responses by increasing transcriptional activity of at least 125 genes, most of which are proinflammatory [25]. Here we used the rat diabetes signaling pathway PCR array to determine key gene expression changes (84 target genes) in the soleus muscle. The PCR array includes genes that contribute to obesity, insulin resistance, the early onset 

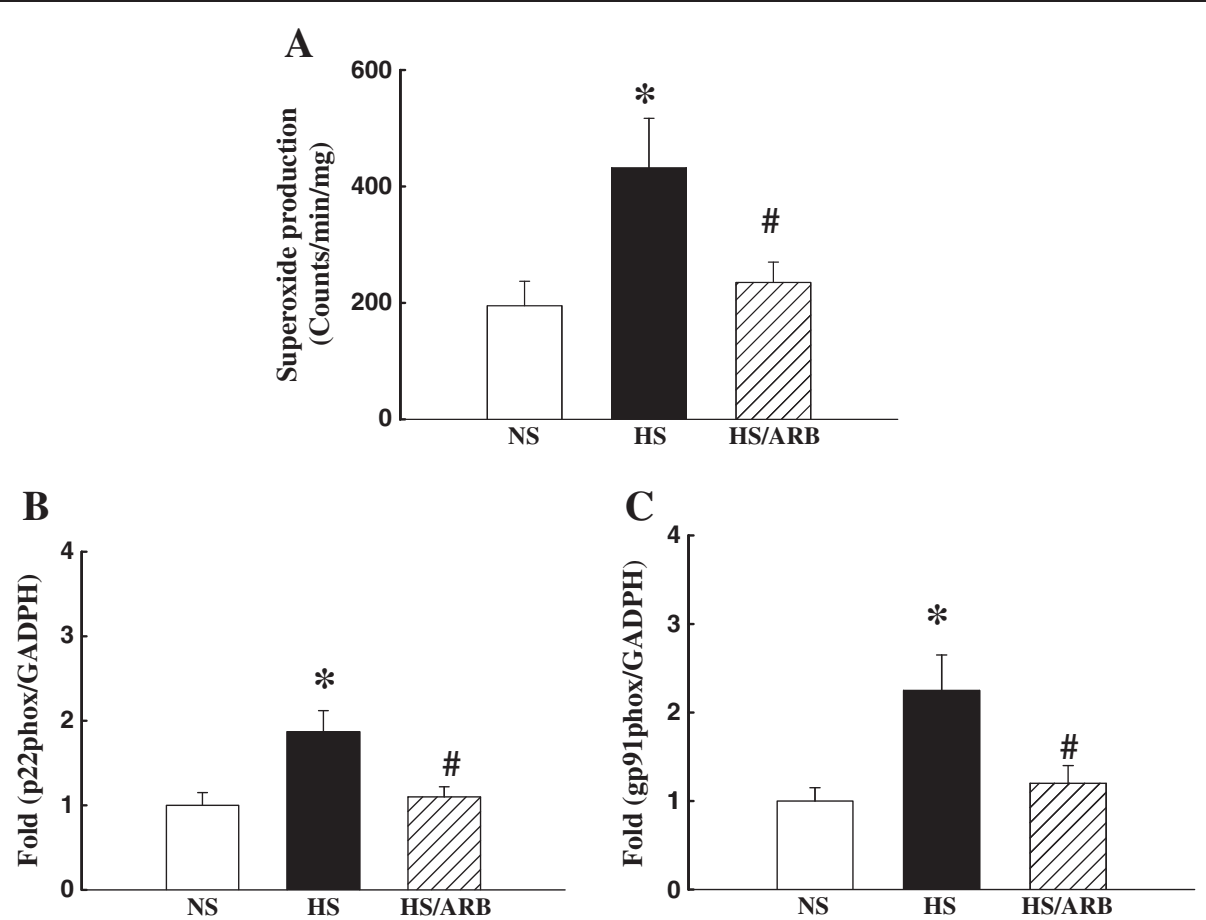

Figure 2 Superoxide $\left(\mathrm{O}_{2}{ }^{-}\right)$production $(\mathbf{A})$ and the mRNA expression of nicotinamide adenine dinucleotide phosphate (reduced form, NADPH) oxidase subunits p22phox (B) and gp91 phox (C) in the soleus of DS rats. $\mathrm{O}_{2}^{-}$production and the expression of p22phox and gp91 phox were significantly increased in the muscle of hypertensive DS rats, compared with normotensive DS rats. ARB candesartan significantly reduced $\mathrm{O}_{2}{ }^{-}$ production and the mRNA expression of p22phox and gp91 phox. ${ }^{*} \mathrm{P}<0.05$ vs. NS, \#p $<0.05$ vs. HS; N $=5-7$.

of diabetes, and complications from diabetes. As shown in the Table 1, among the 84 target genes, 8 genes were altered in the soleus muscle of HS-DS rats treated with control peptide SM-50, compared with NS rats. Of the 8 genes whose expression was significantly altered, 6 increased

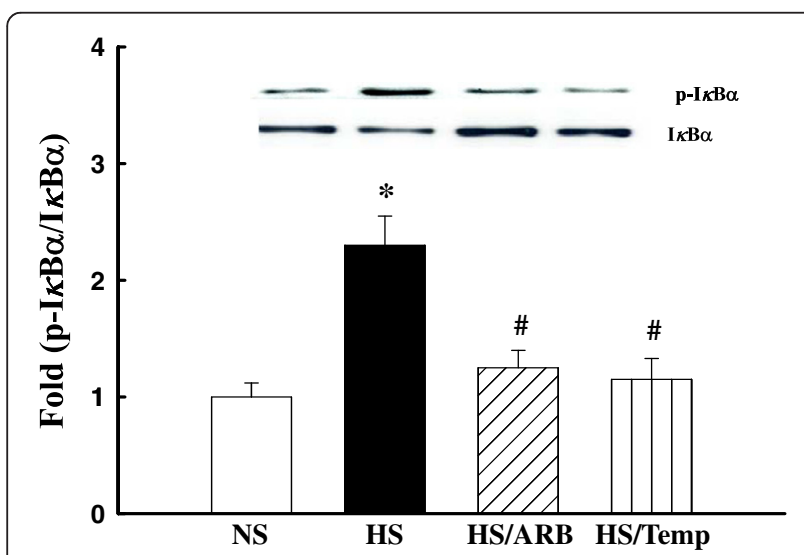

Figure $\mathbf{3}$ Protein expression of inhibitory kappa $\mathrm{Ba}(\mathrm{I} \mathrm{KBa})$ and phospho (Ser32)-Ikba in the soleus muscle of DS rats. The expression of IkBa

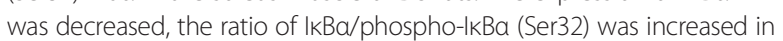
the muscle of hypertensive DS rats, which were significantly inhibited by the treatment of either angiotensin receptor 1 blocker (ARB) candesartan or antioxidant tempol. *P $<0.05$ vs. NS, \#p $<0.05$ vs. HS. $N=4-5$. including: angiotensin converting enzyme (ACE)1, 5 of proinflammatory genes including tumor necrosis factor (TNF) $\alpha$, SOCS3, IL-6, monocyte chemoattractant protein-1 (MCP-1), and intracellular adhesion molecule-1 (ICAM-1). Additionally, 2 genes regulating glucose metabolic

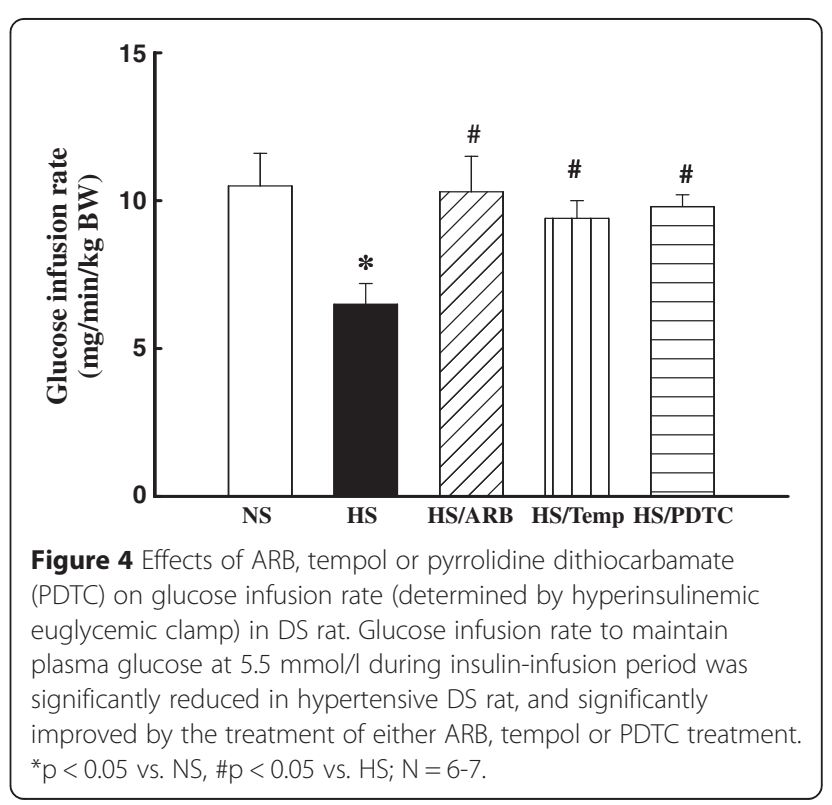




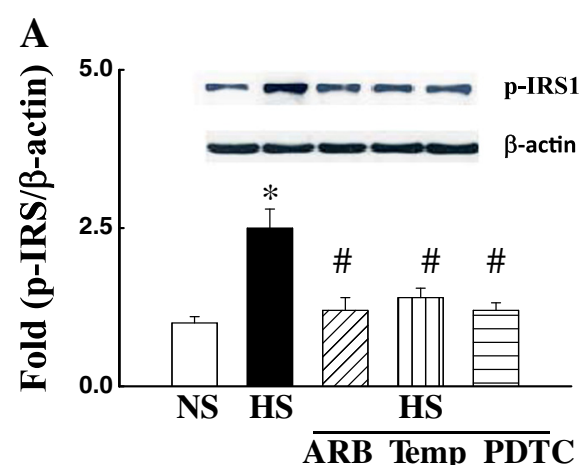

B

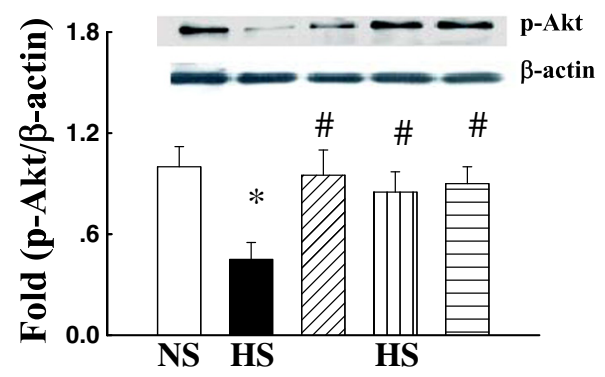

$\overline{\text { ARB Temp PDTC }}$
C

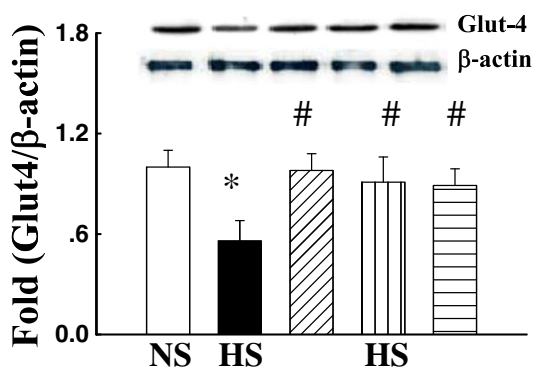

$\overline{\text { ARB Temp PDTC }}$

Figure 5 The protein expression of phospho (Ser 612)-insulin receptor substance 1 (IRS1, A), phospho (Ser 473) Akt (B) and Glut-4 in membrane fraction (C) in the soleus muscle of DS rats. The protein expression of phospho (Ser 612)-IRS1 was increased, and phospho (Ser 473) Akt and Glut-4 in the membrane fraction was significantly decreased in hypertensive DS rats, which was restored by the treatment of either ARB, tempol or PDTC treatment. ${ }^{*} \mathrm{P}<0.05$ vs. NS, \#p $<0.05$ vs. HS; $N=4-5$.

enzyme glycerol-3-phosphate dehydrogenase 1 and peroxisome proliferator-activated receptor coactivator (PCG)1 (the gene that regulate mitochondria biogenesis) were decreased. Pre-incubation with NFkB peptide inhibitor SN50 (HS-SN50) reversed gene expression changes in seven of the above mentioned genes, ACE1 was not significantly affected by SN50 treatment. In addition, we

Table 1 Profile gene by PCR array in skeletal muscle of DS rats (fold increase)

\begin{tabular}{llll}
\hline Gene & NS & HS/SM50 & HS/SN50 \\
\hline ACE1 & $1 \pm 0.1$ & $1.7 \pm 0.2^{*}$ & $1.6 \pm 0.2^{*}$ \\
MCP1 & $1 \pm 0.2$ & $2.5 \pm 0.6^{*}$ & $1.5 \pm 0.4 \#$ \\
ICAM1 & $1 \pm 0.1$ & $8.2 \pm 4.6^{*}$ & $2.9 \pm 1.2^{*} \#$ \\
IL6 & $1 \pm 0.2$ & $10.1 \pm 1.3^{*}$ & $1.7 \pm 1.1^{*} \#$ \\
TNFa & $1 \pm 0.1$ & $1.7 \pm 0.4^{*}$ & $0.7 \pm 0.2 \#$ \\
SOCS3 & $1 \pm 0.1$ & $7.3 \pm 3.6^{*}$ & $1.5 \pm 0.7 \#$ \\
GPD1 & $1 \pm 0.1$ & $0.6 \pm 0.1^{*}$ & $1.1 \pm 0.2 \#$ \\
PGC1 & $1 \pm 0.2$ & $0.4 \pm 0.1^{*}$ & $1.2 \pm 0.2 \#$ \\
\hline
\end{tabular}

Note: PCR: polymerase chain reaction; DS: Dahl salt-sensitive; ACE1: angiotensin converting enzyme 1; MCP1: monocyte chemoattract protein 1; ICAM1: intracellular adhesion molecule 1; IL6: interleukin 6, TNFa; Tumor necrosis factor a; SOCS3: suppressor of cytokine signaling 3; GPD1: Glycerol-3- phosphate dehydrogenase 1, PCG1: PPARy coactivated factor $1 .{ }^{*} \mathrm{p}<0.05$ vs. NS; $\# \mathrm{p}<0.05$ vs. HS-SM50. $\mathrm{N}=4$. further confirmed that individual gene expression of SOCS3 and IL6 was significantly increased in hypertensive rats (HS-SM50) and reduced in hypertensive rats treated with $\mathrm{NFkB}$ inhibitor SN-50 by real-time PCR (Figure 6). These data suggest that activation of $\mathrm{NFKB}$ may play a role in the induction of skeletal muscle inflammation and insulin resistance in hypertension.

\section{Discussion}

The present study demonstrates that the synthesis of Ang II is increased in the skeletal muscle of genetic SS hypertensive DS rats. Increased Ang II stimulation of NADPH oxidase-derived ROS activates NFKB inflammatory pathway, which in turn impairs insulin signaling and Glut-4 translocation in the skeletal muscle and induces systemic insulin resistance.

\section{Association of hypertension and insulin resistance: role of RAS}

Diminished tissue sensitivity to insulin is a central feature of various pathological conditions termed the metabolic syndrome (MS) [1]. Insulin resistance and hypertension are key components of MS and often co-exist. Since patients with MS are commonly afflicted with cardiovascular morbidities, MS and CVDs share common pathways, such 

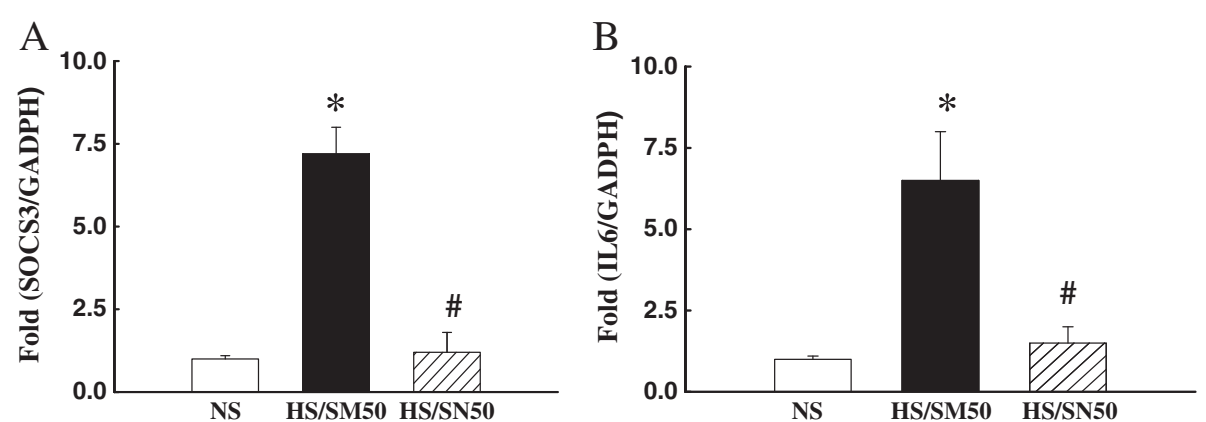

Figure 6 The mRNA expression of suppressors of cytokine signaling 3 (SOCS3, A) and interleukin 6 (IL6, B) was significantly decreased in the soleus muscle of hypertensive DS rats treated with control peptide SM-50. Inhibition of nuclear factor kappa B (NFkB) by specific inhibitor of SN-50 significantly reduced the mRNA expression of SOCS3 or monocyte chemoattractant protein-1 (MCP1). *P $<0.05$ vs. NS, \#p $<0.05$ vs. HS/SM50; N = 4-5.

as, activated RAS, increased oxidative stress, defective glucose and lipid metabolism, low grade inflammation and endothelial damage $[1,2]$.

The effects of the systemic RAS on blood pressure and glucose metabolism have been well demonstrated [9], and local tissue RAS in the skeletal muscle, vasculature, adipocytes and pancreas may also play an important role in the development of insulin resistance and vascular injury in diabetic and hypertensive diseases $[14,26]$. Ang II (via AT1R), the predominant component of RAS, induces insulin resistance through variety of mechanisms including inhibition of insulin signaling and insulin mediated glucose uptake in the skeletal muscle, decreased insulin secretion from pancreatic beta cells and alternation in adipocyte homeostasis $[27,28]$. In contrast to the Ang II/AT1R axis, other components of RAS, such as Ang (1-7), AT2R or AT4R may have alternate effects on insulin sensitivity and vascular function [29-31]. For example, Ang- (1-7) improves insulin sensitivity and pancreatic $\beta$ cell survival in STZ-induced diabetic mice [31] and deletion of AT2R may reverse diabetes-induced endothelial function and vascular injury [30]. We have previously shown that in SS hypertension upregulation of local Ang II action (vasculature) induced endothelial dysfunction and vascular injury [13], here we further demonstrated that the blockade of Ang II by ARB candesartan improved skeletal muscle and systemic insulin sensitivity in hypertensive DS rats. Our data support the idea that activation of RAS is a common link between insulin resistance and vascular injury in SS hypertension.

We have to emphasize although it has been shown that ARBs are superior to other active antihypertensive agents for reduced incidence of new onset of TD2M in the patients with hypertension, the association of ARBs with diabetes risk may differ [32,33]. Some ARBs, for example, olmesartan may be associated with a slightly increased risk of diabetes mellitus [32]. The different actions of antidiabetic effects by ARBs may be related to improvement of insulin sensitivity and other properties of ARBs such as activation of peroxisome proliferator-activated receptor gamma or insulin sensitizing effects [33,34].

\section{RAS/ROS in skeletal muscle insulin resistance}

It has been shown that skeletal muscle cells contain many components of RAS, including angiotensinogen, Ang I and Ang II, ACE1, AT1R and AT2R [21,35]. The present study showed that Ang II content was increased in the skeletal muscle of hypertensive DS rats. As the expression of ACE1 was also increased in the skeletal muscle of hypertensive DS rats (Table 1), the increased Ang II content may mainly be due to an increased rate of in situ synthesis of Ang II. Therefore, Ang II has prooxidant effects, as the upregulation of Ang II increased ROS production through stimulation of NADPH oxidase in the muscle (Figure 2) [36].

An increasing body of evidence supports the role of Ang II in the multifactorial etiology of skeletal muscle insulin resistance [36-38]. Acute infusion of Ang II into the interstitial space of skeletal muscle has been shown to impair insulin-mediated glucose uptake, which was independent of alteration in blood flow [39]. Chronic infusion of Ang II in the rat was associated with diminution of whole body glucose disposal and reduced skeletal muscle glucose uptake, likely due to increased ROS production $[3,40]$. Furthermore, in cultured L6 myotubes, Ang II stimulated serine-phosphorylation of IRS-1 [36]. Phosphorylation of IRS-1 at specific serine residues inhibited insulin stimulation of tyrosine phosphorylation, subsequently inhibiting downstream PI3K signaling [14].

The present study demonstrated that upregulation of endogenous Ang II-induced ROS impaired insulin signaling and Glut-4 translocation in the skeletal muscle of hypertensive rats and resulted in systemic insulin resistance. Since Ang II/ROS (via NADPH oxidase) is present in both skeletal myocytes and the vascular tissue of arterioles that supply blood to these myocytes $[26,35]$, a 
large number of studies have shown a relationship between microvascular dysfunction and skeletal muscle insulin resistance [41-43]. The present study has some limitations that we did not investigate the effects of insulin on microvascular function in the skeletal muscle. However, based on our previous findings that ARB or antioxidant tempol improved endothelium function and insulin-induced vasorelaxation in the aorta of this animal model $[13,15]$, it is reasonable to speculate that improvement of skeletal muscle insulin resistance by the ARB or antioxidant tempol may be, at least in part, attributed to their hemodynamic action in skeletal muscle $[26,36,40]$.

\section{Ang II and ROS converge on NFKB to inhibit insulin signaling}

It is increasingly recognized that chronic inflammation plays a critical role in the pathogenesis of hypertension, insulin resistance and atherosclerotic diseases [44,45]. $\mathrm{NFKB}$ is a redox-sensitive transcript factor that regulates the transcription of a large number of proinflammatory genes. Ang II, oxidative stress and proinflammatory cytokines are the factors that induce $\mathrm{NF \kappa B}$ activation [25]. Ang II binding to AT1R activates NFkB in a ROSdependent manner. ROS activates IKB kinase (IKK) to induce ІкB phosphorylation, resulting in the activation of NFkB $[25,46]$. Here we showed that hypertensive DS rats exhibited a decreased I $\mathrm{K} B$ and increased the ratio of phospho-IкB $\alpha / \mathrm{I} \kappa \mathrm{B} \alpha$, which was prevented by either ARB candesartan or antioxidant tempol, suggesting involvement of Ang II/ROS in activation of NFKB in skeletal muscle of this animal model. Furthermore, inhibition of $\mathrm{NFKB}$ using SN50 reversed gene profile related to development of insulin resistance and diabetes and improved skeletal muscle insulin signaling. Moreover, a number of the genes such as proinflammatory genes of TNF $\alpha$, IL6 and COCS3, which were increased in the muscle of hypertensive DS rats (Table 1), have been shown to play a fundamental role in the pathogenesis of insulin resistance and T2DM $[47,48]$. Therefore, our data support the idea that in SS hypertension, Ang II and ROS converge on $\mathrm{NF \kappa B}$ signaling, which in turn induces skeletal muscle inflammation and insulin resistance.

In summary, clinical and experimental studies have shown that the inhibition of Ang II by ACE inhibitors or ARBs or antioxidants improve insulin sensitivity and glycemic control in diabetic patients and animals and reduce the incidence of new onset type 2 diabetes $[33,39]$. The present study demonstrated that increased endogenous Ang II/ROS activation of NFKB inflammatory pathway impairs insulin signaling and Glut-4 transolcation in the skeletal muscle of SS hypertension. The current finding, in conjunction with our recent studies $[13,15]$ in the effects of ARB, antioxidant or NFkB inhibition on endothelial function, vascular inflammation and
CV injury strongly suggest that Ang II/ROS activation of $\mathrm{NF \kappa B}$ is an important link among hypertension, vascular injury and insulin resistance.

\section{Abbreviation}

Ang II: Angiotensin II; ACE: Angiotensin converting enzyme; ARB: Angiotensin receptor 1 blocker; CVD: Cardiovascular disease; DS: Dahl salt-sensitive; IRS1: Insulin receptor substance 1; IL6: Interleukin 6; ICAM1: Intracellular adhesion molecule-1; MAP: Mean arterial blood pressure; MCP-1: Monocyte chemoattractant protein-1; NADPH: Nicotinamide adenine dinucleotide phosphate (reduced form); NO: Nitric oxide; NFkB: Nuclear factor kappa B; PI3K: Phosphatidylinositol 3-kinase; PDTC: Pyrrolidine dithiocarbamate; ROS: Reactive oxygen species; RAS: Renin-angiotensin system; SS: Saltsensitive; $\mathrm{O}_{2}^{-}$: Superoxide anion; SOCS3: Suppressors of cytokine signaling 3; TNFa: Tumor necrosis factor a; T2DM: Type 2 diabetic mellitus.

\section{Competing interests}

The authors declare that they have no competing interests.

\section{Authors' contributions}

Participated in research design: MSZ, and LR; conducted experiments: MSZ, $\mathrm{CL}, \mathrm{AN}$ and RXT; all authors performed data analysis and interpretation; MSZ and LR contributed to the writing of the manuscript: all authors read and approved the final manuscript.

\section{Acknowledgement}

This work was supported by the grant from the National Natural Science Foundation of China (No. 81470532) and American Heart Association National Scientist Development Award to Ming-Sheng Zhou, and by funds from the South Florida Veterans Affairs Foundation for Research and Education to Leopoldo Raij.

\section{Author details}

${ }^{1}$ Department of Physiology, Liaoning Medical University, Jinzhou, China. ${ }^{2}$ Department of Endocrinology, Liaoning Medical University, Jinzhou, China. ${ }^{3}$ Hypertension/Nephrology Section, Miami VA Medical Center, Miami, FL, USA. ${ }^{4}$ Department of Pharmacology, Kagawa University School of Medicine, Kagawa, Japan. ${ }^{5}$ Hypertension/Nephrology section, Vascular Biology Institute, University of Miami Miller School of Medicine, Miami, FL, USA.

Received: 11 February 2015 Accepted: 10 April 2015

Published online: 01 May 2015

\section{References}

1. Zhou MS, Wang A, Yu H. Link between insulin resistance and hypertension: What is the evidence from evolutionary biology? Diabetol Metab Syndr. 2014;6(1):12.

2. Ferrannini E, Cushman WC. Diabetes and hypertension: the bad companions. Lancet. 2012;380(9841):601-10.

3. Lastra-Lastra G, Sowers JR, Restrepo-Erazo K, Manrique-Acevedo C, Lastra-Gonzalez G. Role of aldosterone and angiotensin II in insulin resistance: an update. Clin Endocrinol (Oxf). 2009;71(1):1-6.

4. Chen J, Gu D, Huang J, Rao DC, Jaquish CE, Hixson JE, et al. Metabolic syndrome and salt sensitivity of blood pressure in non-diabetic people in China: a dietary intervention study. Lancet. 2009;373(9666):829-35.

5. Stolarz-Skrzypek K, Kuznetsova T, Thijs L, Tikhonoff V, Seidlerova J, Richart T, et al. Fatal and nonfatal outcomes, incidence of hypertension, and blood pressure changes in relation to urinary sodium excretion. JAMA. 2011;305(17):1777-85.

6. Lastra G, Dhuper S, Johnson MS, Sowers JR. Salt, aldosterone, and insulin resistance: impact on the cardiovascular system. Nat Rev Cardiol. 2010;7(10):577-84.

7. Zhou MS, Schulman $\mathbb{H}$, Zeng Q. Link between the renin-angiotensin system and insulin resistance: implications for cardiovascular disease. Vasc Med. 2012;17(5):330-41.

8. Van Gaal LF, Mertens IL, De Block CE. Mechanisms linking obesity with cardiovascular disease. Nature. 2006;444(7121):875-80.

9. Cooper SA, Whaley-Connell A, Habibi J, Wei Y, Lastra G, Manrique C, et al. Renin-angiotensin-aldosterone system and oxidative stress in cardiovascular insulin resistance. Am J Physiol Heart Circ Physiol. 2007;293(4):H2009-23. 
10. Holland WL, Bikman BT, Wang LP, Yuguang G, Sargent KM, Bulchand S, et al. Lipid-induced insulin resistance mediated by the proinflammatory receptor TLR4 requires saturated fatty acid-induced ceramide biosynthesis in mice. J Clin Invest. 2011;121(5):1858-70.

11. Steinberg HO, Brechtel G, Johnson A, Fineberg N, Baron AD. Insulin-mediated skeletal muscle vasodilation is nitric oxide dependent. A novel action of insulin to increase nitric oxide release. J Clin Invest. 1994;94(3):1172-9.

12. Folli F, Kahn CR, Hansen $H$, Bouchie $J$, Feener EP. Angiotensin II inhibits insulin signaling in aortic smooth muscle cells at multiple levels. A potential role for serine phosphorylation in insulin/angiotensin II crosstalk. J Clin Invest. 1997;100(9):2158-69.

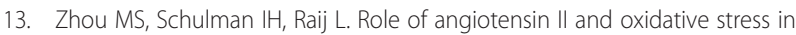
vascular insulin resistance linked to hypertension. Am J Physiol Heart Circ Physiol. 2009;296(3):H833-9.

14. Andreozzi F, Laratta E, Sciacqua A, Perticone F, Sesti G. Angiotensin II impairs the insulin signaling pathway promoting production of nitric oxide by inducing phosphorylation of insulin receptor substrate-1 on Ser312 and Ser616 in human umbilical vein endothelial cells. Circ Res. 2004;94(9):1211-8.

15. Zhou MS, Schulman $I H$, Raij L. Vascular inflammation, insulin resistance, and endothelial dysfunction in salt-sensitive hypertension: role of nuclear factor kappa B activation. J Hypertens. 2010;28(3):527-35.

16. Zhou MS, Hernandez Schulman I, Pagano PJ, Jaimes EA, Raij L. Reduced $\mathrm{NAD}(\mathrm{P}) \mathrm{H}$ oxidase in low renin hypertension: link among angiotensin II, atherogenesis, and blood pressure. Hypertension. 2006;47(1):81-6.

17. Kiechl S, Wittmann J, Giaccari A, Knoflach M, Willeit P, Bozec A, et al. Blockade of receptor activator of nuclear factor-kappaB (RANKL) signaling improves hepatic insulin resistance and prevents development of diabetes mellitus. Nat Med. 2013;19(3):358-63.

18. Reilly SM, Chiang SH, Decker SJ, Chang L, Uhm M, Larsen MJ, et al. An inhibitor of the protein kinases TBK1 and IKK-varepsilon improves obesityrelated metabolic dysfunctions in mice. Nat Med. 2013;19(3):313-21.

19. Vandanmagsar B, Youm YH, Ravussin A, Galgani JE, Stadler K, Mynatt RL, et al. The NLRP3 inflammasome instigates obesity-induced inflammation and insulin resistance. Nat Med. 2011;17(2):179-88.

20. Cai D, Yuan M, Frantz DF, Melendez PA, Hansen L, Lee J, et al. Local and systemic insulin resistance resulting from hepatic activation of IKK-beta and NF-kappaB. Nat Med. 2005;11(2):183-90.

21. Stump CS, Henriksen EJ, Wei Y, Sowers JR. The metabolic syndrome: role of skeletal muscle metabolism. Ann Med. 2006;38(6):389-402.

22. Nishiyama A, Yoshizumi M, Rahman M, Kobori H, Seth DM, Miyatake A, et al. Effects of AT1 receptor blockade on renal injury and mitogen-activated protein activity in Dahl salt-sensitive rats. Kidney Int. 2004;65(3):972-81.

23. Zhou MS, Adam AG, Jaimes EA, Raij L. In salt-sensitive hypertension, increased superoxide production is linked to functional upregulation of angiotensin II. Hypertension. 2003;42(5):945-51.

24. Arkan MC, Hevener AL, Greten FR, Maeda S, Li ZW, Long JM, et al. IKK-beta links inflammation to obesity-induced insulin resistance. Nat Med. 2005;11(2):191-8.

25. Ghosh S, Hayden MS. New regulators of NF-kappaB in inflammation. Nat Rev Immunol. 2008;8(11):837-48.

26. Underwood PC, Adler GK. The renin angiotensin aldosterone system and insulin resistance in humans. Curr Hypertens Rep. 2013;15(1):59-70.

27. Wei Y, Chen K, Whaley-Connell AT, Stump CS, Ibdah JA, Sowers JR. Skeletal muscle insulin resistance: role of inflammatory cytokines and reactive oxygen species. Am J Physiol Regul Integr Comp Physiol. 2008;294(3):R673-80.

28. Chu KY, Lau T, Carlsson PO, Leung PS. Angiotensin II type 1 receptor blockade improves beta-cell function and glucose tolerance in a mouse model of type 2 diabetes. Diabetes. 2006;55(2):367-74.

29. Liu M, Jing D, Wang Y, Liu Y, Yin S. Overexpression of angiotensin II type 2 receptor promotes apoptosis and impairs insulin secretion in rat insulinoma cells. Mol Cell Biochem. 2015;400(1-2):233-44.

30. Nasser M, Clere N, Botelle L, Javellaud J, Oudart N, Faure S, et al. Opposite effects of angiotensins receptors type 2 and type 4 on streptozotocin induced diabetes vascular alterations in mice. Cardiovasc Diabetol. 2014;13:40.

31. He J, Yang Z, Yang H, Wang L, Wu H, Fan Y, et al. Regulation of insulin sensitivity, insulin production, and pancreatic beta cell survival by angiotensin-(1-7) in a rat model of streptozotocin-induced diabetes mellitus. Peptides. 2015;64C:49-54.

32. Chang CH, Chang YC, Wu LC, Lin JW, Chuang LM, Lai MS. Different angiotensin receptor blockers and incidence of diabetes: a nationwide population-based cohort study. Cardiovasc Diabetol. 2014;13:91.
33. Abuissa $\mathrm{H}$, Jones PG, Marso SP, O'Keefe Jr JH. Angiotensin-converting enzyme inhibitors or angiotensin receptor blockers for prevention of type 2 diabetes: a meta-analysis of randomized clinical trials. J Am Coll Cardiol. 2005;46(5):821-6.

34. Schupp M, Janke J, Clasen R, Unger T, Kintscher U. Angiotensin type 1 receptor blockers induce peroxisome proliferator-activated receptor-gamma activity. Circulation. 2004;109(17):2054-7.

35. Jones A, Woods DR. Skeletal muscle RAS and exercise performance. Int J Biochem Cell Biol. 2003;35(6):855-66.

36. Wei Y, Sowers JR, Clark SE, Li W, Ferrario CM, Stump CS. Angiotensin II-induced skeletal muscle insulin resistance mediated by NF-kappaB activation via NADPH oxidase. Am J Physiol Endocrinol Metab. 2008;294(2):E345-51.

37. Wang N, Chai W, Zhao L, Tao L, Cao W, Liu Z. Losartan increases muscle insulin delivery and rescues insulin's metabolic action during lipid infusion via microvascular recruitment. Am J Physiol Endocrinol Metab. 2013;304(5):E538-45.

38. Pan Y, Qiao QY, Pan LH, Zhou DC, Hu C, Gu HF, et al. Losartan reduces insulin resistance by inhibiting oxidative stress and enhancing insulin signaling transduction. Exp Clin Endocrinol Diabetes. 2015;123(3):170-7.

39. Putnam K, Shoemaker R, Yiannikouris F, Cassis LA. The renin-angiotensin system: a target of and contributor to dyslipidemias, altered glucose homeostasis, and hypertension of the metabolic syndrome. Am J Physiol Heart Circ Physiol. 2012;302(6):H1219-30.

40. Wei Y, Sowers JR, Nistala R, Gong H, Uptergrove GM, Clark SE, et al. Angiotensin Il-induced NADPH oxidase activation impairs insulin signaling in skeletal muscle cells. J Biol Chem. 2006;281(46):35137-46.

41. De Boer MP, Meijer RI, Wijnstok NJ, Jonk AM, Houben AJ, Stehouwer CD, et al. Microvascular dysfunction: a potential mechanism in the pathogenesis of obesity-associated insulin resistance and hypertension. Microcirculation. 2012;19(1):5-18.

42. Clark MG. Impaired microvascular perfusion: a consequence of vascular dysfunction and a potential cause of insulin resistance in muscle. Am J Physiol Endocrinol Metab. 2008;295(4):E732-50.

43. Bonner JS, Lantier L, Hasenour CM, James FD, Bracy DP, Wasserman DH. Musclespecific vascular endothelial growth factor deletion induces muscle capillary rarefaction creating muscle insulin resistance. Diabetes. 2013;62(2):572-80.

44. Sriwijitkamol A, Christ-Roberts C, Berria R, Eagan P, Pratipanawatr T, DeFronzo RA, et al. Reduced skeletal muscle inhibitor of kappaB beta content is associated with insulin resistance in subjects with type 2 diabetes: reversal by exercise training. Diabetes. 2006;55(3):760-7.

45. Lu J, Ji J, Meng H, Wang D, Jiang B, Liu L, et al. The protective effect and underlying mechanism of metformin on neointima formation in fructoseinduced insulin resistant rats. Cardiovasc Diabetol. 2013;12:58.

46. Ogihara T, Asano T, Katagiri H, Sakoda H, Anai M, Shojima N, et al. Oxidative stress induces insulin resistance by activating the nuclear factor-kappa B pathway and disrupting normal subcellular distribution of phosphatidylinositol 3-kinase. Diabetologia. 2004;47(5):794-805.

47. Jiang Y, Thakran S, Bheemreddy R, Ye EA, He H, Walker RJ, et al. Pioglitazone normalizes insulin signaling in the diabetic rat retina through reduction in tumor necrosis factor alpha and suppressor of cytokine signaling 3. J Biol Chem. 2014;289(38):26395-405.

48. De Filippo G, Rendina D, Moccia F, Rocco V, Campanozzi A. Interleukin-6, soluble interleukin-6 receptor/interleukin- 6 complex and insulin resistance in obese children and adolescents. J Endocrinol Invest. 2014; Sep 23. [Epub ahead of print].

\section{Submit your next manuscript to BioMed Central and take full advantage of:}

- Convenient online submission

- Thorough peer review

- No space constraints or color figure charges

- Immediate publication on acceptance

- Inclusion in PubMed, CAS, Scopus and Google Scholar

- Research which is freely available for redistribution 